\title{
10 Jahre Hauttumor-Workshop in Dresden - ein wissenschaftlicher Höhepunkt zur Verabschiedung von Professor Günther Sebastian
}

\author{
$10^{\text {th }}$ Skin Cancer Workshop in Dresden - A Scientific Highlight Upon Retirement of Prof. Guenther Sebastian
}

\section{Autor}

Institut

\section{U. Proske}

Klinik und Poliklinik für Dermatologie, Medizinische Fakultät der Technischen Universität Dresden

\section{Bibliografie}

Dol $10.1055 / \mathrm{s}-2007-966085$

Akt Dermatol 2007; 33;

47-48 @ Georg Thieme

Verlag KG Stuttgart · New York ISSN 0340-2541

Korrespondenzadresse

Dr. med. Ulrike Proske Klinik und Poliklinik für Dermatologie, Medizinische Fakultät der Technischen Universität Dresden

Fetscherstraße 74 01307 Dresden Ulrike.Proske@uniklinikumdresden.de
In diesem Jahr trafen 3 wichtige Jubiläen in Dresden aufeinander. Im Jahr 2006 jährte sich die Stadtgründung Dresdens zum 800., der Geburtstag von Prof. Sebastian zum 65. und der Hauttumor-Workshop zum 10. Male. Diese Ereignisse boten einen würdigen Rahmen für die Veranstaltung des Workshops, der dank des jährlich wachsenden Interesses wieder mit reger Beteiligung durchgeführt wurde. Adressiert ist er an alle onkologisch tätigen Dermatologen aus Kliniken und Niederlassung, wobei wir in diesem Jahr 150 Teilnehmer aus allen „Anrainerländern“ Sachsens, also Bayern, Thüringen, Sachsen-Anhalt und Brandenburg begrüßen durften. Die Tagung fand am 15. und 16. September 2006 in Dresden in den Räumlichkeiten der Berufsgenossenschaftlichen Akademie für Arbeitssicherheit und Gesundheitsschutz, einem von Tessenow und Kramer erbauten ehemaligen Schulgelände, statt. Damit verbindet sich die 800 -jährige Geschichte der Stadt mit der avantgardistischen Architektur der 20er Jahre mit Bauhausgedanken.

Die Thematik „Innovationen in der Prävention, Diagnostik und Therapie bei Präkanzerosen und häufigen Hauttumoren“ entspricht den Arbeitsschwerpunkten von Prof. Günther Sebastian und war ein Garant für eine abwechslungsreiche Tagung. Die weitgespannte Thematik wurde von klinisch und wissenschaftlich ausgewiesenen Experten unseres Faches referiert.

Am ersten Tag stand das primäre Melanom im Vordergrund. Die Möglichkeiten der operativen Versorgung wurden von R. Rompel (Kassel) an anschaulichen Beispielen dargelegt. Die SentinelLymphknotenbiopsie (SLND), seit längerer Zeit beim malignen Melanom eingeführt, bietet immer noch viel Diskussionsstoff. Ihre Bedeutung als exakte Staging-Methode und prognostischer Faktor ist unbestritten, allerdings sollten die Schwierigkeiten der Detektion an besonderen Lokalisationen wie z. B. Hals oder Axilla berücksichtigt werden (J. Koller, Salzburg).

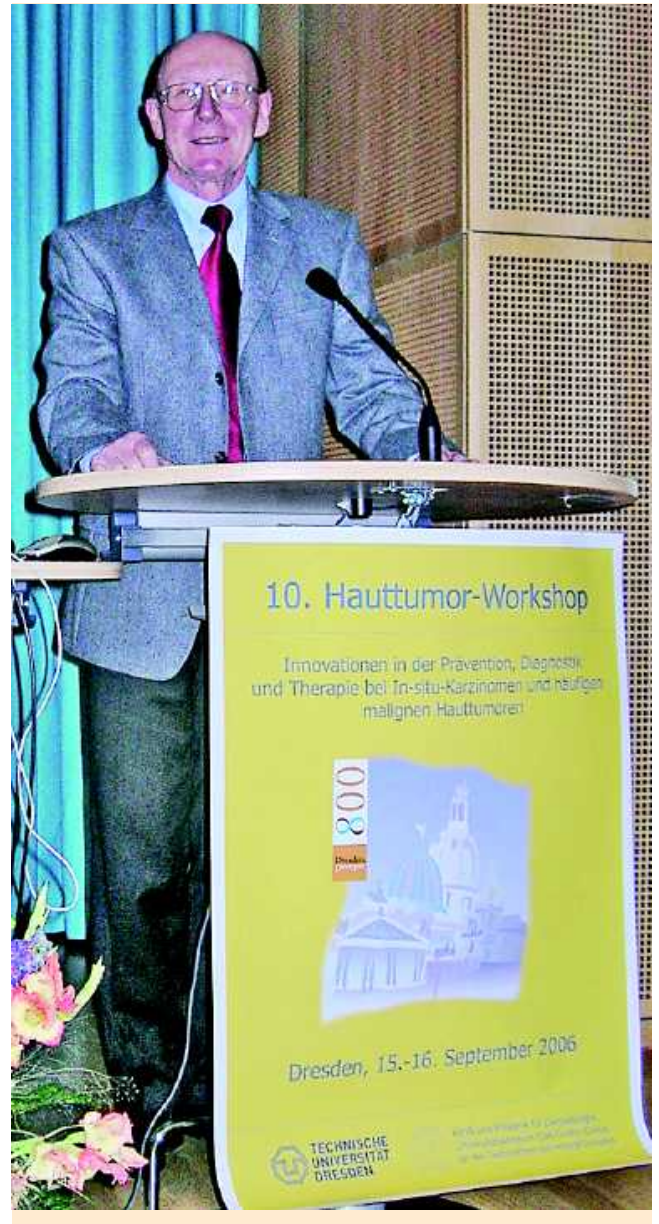

Abb. 1 Prof. G. Sebastian bei der Eröffnung des 10. Hauttumor-Workshops.

A. Hauschild (Kiel) unterstrich die Bedeutung von Interferon alpha in der adjuvanten Melanom-Therapie, da es die erste Substanz ist, die in prospektiv randomisierten Studien zu einem signifikanten Vorteil für die Behandelten geführt hat. Er stellte neue Substanzen vor, bei denen die Behandlungsergebnisse an größeren Patienten- 


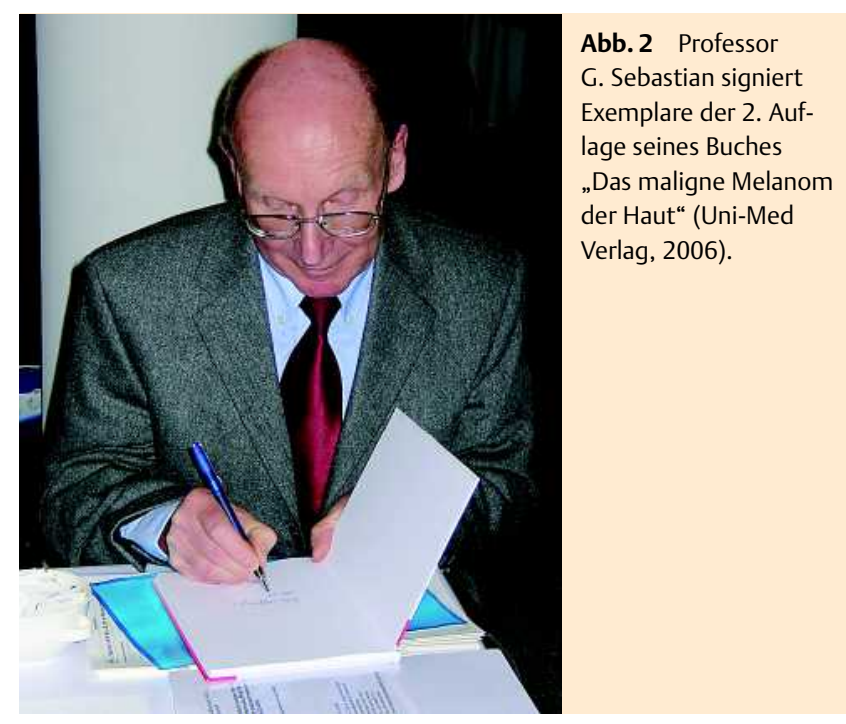

kollektiven und im Rahmen von Studien mit Spannung erwartet werden. W. Tilgen (Homburg/S.) referierte über neue Entwicklungen in der palliativen Melanomtherapie. Die Ergebnisse der Chemo-/Chemoimmuntherapie im Stadium einer systemischen Metastasierung sind ernüchternd. Die therapeutischen Bemühungen sollten unbedingt die Lebensqualität des Patienten berücksichtigen, da die Prognose im Stadium der Fernmetastasierung weiterhin infaust ist. Sichtbare und funktionell einschränkende Hautmetastasen stellen bei Patienten mit metastasiertem malignem Melanom ein besonderes therapeutisches Problem dar. S. Eppinger (Dresden) zeigte eine Vielzahl von Behandlungsmöglichkeiten, deren Anwendungsmodalitäten und Erfolge für den Einzelfall auf. Wenn chirurgische Interventionen nicht möglich sind, stehen Radiatio, Lasertherapie und verschiedene Lokaltherapien zur Verfügung, wobei die Therapieentscheidung stets der individuellen Situation und dem Allgemeinzustand des Patienten angepasst werden muss.

Über die Organisation der Melanomnachsorge, die Häufigkeit und den Umfang der Untersuchungen sprach Frau R. Linse (Erfurt). Besonders wichtig ist dabei die Zusammenarbeit der Kliniken mit den niedergelassenen Fachärzten, um für den Patienten eine sinnvolle Versorgung zu garantieren. Die Besonderheiten eines onkologischen Netzwerkes betonte U. Reinhold (Bonn). Schwerpunkte des Netzwerkes sind die Einleitung bzw. Fortführung der Tumortherapie, Verlaufskontrolle und individuelle Rehabilitation in der Praxis beim niedergelassenen Dermatologen mit onkologischem Schwerpunkt.

Am zweiten Tag standen die Erkrankungen an lichtgeschädigter Haut im Vordergrund. Die aktinische Keratose ist ein in hellhäutiger Bevölkerung häufig als sog. „Feldkanzerisierung“ sichtbarer Lichtschaden. Eine heftige Diskussion entzündete sich an der Frage, ob es sich bei dieser Hautveränderung bereits um ein Insitu-Karzinom handelt. C. Garbe (Tübingen) bezog sich bei der Beantwortung dieser Frage auf die Tatsache, nach der die aktinische Keratose eine intraepitheliale Neoplasie mit Aufhebung der normal geschichteten Epidermis ist, verbunden mit dem Auftreten zellulärer Atypien. Bereits am Vortage hatte Th. Schwarz (Kiel) sehr anschaulich über die Mechanismen der UV-induzierten Karzinogenese berichtet. Das Spektrum der primär operativen Verfahren stellte U. Hohenleutner (Regensburg) dar, über die vielfältigen Möglichkeiten der topischen Therapie referierte Th. Dirschka (Wuppertal) und den Standort der photodynami- schen Therapie im therapeutischen Angebot erläuterte $R$. Aschoff (Dresden). Ein Sonderfall ist die aktinische Cheilitis als therapeutische Problemlokalisation. Von I. Hackert und A. Stein (Dresden) wurden verschiedene Behandlungsoptionen kritisch gegeneinander abgewogen. J. Petres (Freiburg) erläuterte an Beispielen die korrektiven Maßnahmen bei epithelialen Gesichtstumoren und hob neben dem primär kurativen den kosmetischen Anspruch unseres Fachgebietes hervor. Besonders häufig treten die nichtmelanozytären Hauttumoren bei immunsupprimierten Patienten, z.B. nach Organtransplantation, auf. Bekannt ist neben der massiv erhöhten Inzidenz der Plattenepithelkarzinome der oft rasante und ungünstige Verlauf. K. Schäkel (Dresden) erläuterte die besonderen Mechanismen der Tumorentstehung in diesem Patientenkollektiv, die an verschiedene individuelle Faktoren wie z. B. höheres Alter und Langzeitimmunsuppression gekoppelt sind.

Da G. Sebastian bis 2006 vier Jahre als Präsident der Vereinigung für Operative und Onkologische Dermatologie (VOD) sehr engagiert tätig war, lag es nahe, den Belangen der VOD auf dieser Tagung Raum zu geben. Frau D. Dill-Müller (Homburg/S.) erläuterte die Zukunftsvisionen der VOD, wobei ihr besonders die Austauschprogramme für operativ interessierte Ärzte zur Unterstützung der Ausbildung am Herzen lagen.

Am Nachmittag demonstrierte Frau U. Boashie (Dresden) die Ergebnisse einer Untersuchung zur kumulativen UV-Belastung verschiedener Körperregionen. Dabei wurde bestätigt, dass sich Büroarbeiter während ihrer Freizeitaktivitäten einer vielfach höheren UV-Dosis als Freilandarbeiter aussetzen. Hier werden Reserven in der Aufklärungsarbeit sichtbar. Das Auftreten von malignen Tumoren der Haut nach kombiniertem Kontakt mit Arsen und uranhaltigem Gestein ist eine berufsdermatologisch relevante Thematik im Wismut-Bergbau, die J. Meyer (Gera) als erfahrener Gutachter erläuterte. Zum Thema, wann UV-induzierte Hauttumore als Berufserkrankungen anerkannt werden können, referierte K. Scheuch (Dresden). Zu dieser Fragestellung ist noch kein abschließender Konsens gefunden, individuelle Lösungen sind jedoch weiterhin anzustreben. Damit wurde der Bogen zu den Präventionsmaßnahmen geschlagen, über die U. Reinhold (Bonn) abschließend referierte. Kritisch und detailliert wurden die Effekte durch Radikalfänger, Retinoide, Karotinoide und NSAR aufgezeigt.

Ein besonderer Höhepunkt war die Abendveranstaltung mit einem sehr stimmungsvollen Konzert in der Dresdener Frauenkirche.

Neben den interessanten und breit gefächerten wissenschaftlichen Themen bot der Workshop auch einen würdigen Rahmen zum Abschluss der beruflichen Tätigkeit von Professor Günther Sebastian. Er kann auf 39 Jahre Berufstätigkeit an der Klinik und Poliklinik für Dermatologie in Dresden zurückblicken, in denen er mit außerordentlichem Engagement die Belange der operativen und onkologischen Dermatologie im In- und Ausland vertreten hat. Er war dabei mehreren Generationen von Lernenden ein hoch motivierter Lehrer, der fachliche Kompetenzen mit menschlicher Zuwendung und Anteilnahme paarte. Er wusste mitzureißen und zu begeistern, was sich auch an der großen Zahl der Gratulanten bewies.

Wir wünschen dem Hauttumor-Workshop weiterhin regen $\mathrm{Zu}$ spruch und Professor Sebastian Kraft und Energie für neue Aufgaben! 